\title{
NATURE POPULAIRE CONTRE NATURE SAVANTE? RENCONTRE ENTRE PÊCHEURS AU CARRELET ET GESTIONNAIRES AUTOUR DE L'ALOSE DU RHÔNE.
}

\author{
C. BARTHÉLÉMY
}

ESA-CNRS 5023, équipe DESMID, 1 rue Parmentier, 13200 Arles, France.

RÉSUMÉ

Depuis une dizaine d'années, l'alose du Rhône fait l'objet d'une politique de restauration. Celle-ci s'inscrit dans un plan national de gestion des poissons migrateurs, réagissant à la raréfaction de ces espèces. Ces nouveaux gestionnaires se heurtent parfois à lincompréhension ou à la non-coopération d'une population, pourtant très intéressée par ces actions, à savoir les pêcheurs amateurs. Une enquête sociologique réalisée dans la région du Bas-Rhône auprès des pêcheurs au carrelet et de l'association en charge de la gestion piscicole des aloses peut permettre de comprendre ces différents. Elle montre, en effet, les difficultés de reconnaissance entre ces deux groupes sociaux qui ont une pratique et une représentation de la nature divergentes. Cet exemple pose au sociologue la question de la légitimité de ces nouvelles politiques de gestion des milieux piscicoles par rapport à des usages plus traditionnels des espaces concernés.

Mots-clés : pêche amateur, gestion piscicole, alose, Rhône, sociologie.

\section{POPULAR NATURE AGAINST SCIENTIST NATURE? AN ENCOUNTER BETWEEN AMATEUR FISHERS AND ADMINISTRATORS AROUND THE RHÔNE'S SHAD.}

\section{ABSTRACT}

Since ten years, the Rhône's shad is the object of a political of restoration. This one is in keeping with a national programme of migratory fishes administration, to react against the rarefaction of these species. Sometimes, these new administrators come up against the incomprehension or a lack of cooperation from a population, nevertheless interesting by these actions, the amateur fishers. A sociological study realised in the area of the lower-Rhone with tishers using a net called "carrelet " and with the association who administrate the shad's migration can explain these disagreements. It reveals the difficult recognition between two social groups who have different practices and representations of nature. This example questions the sociologist about the legitimacy of new administration in relation with some more traditional uses of these spheres.

Key-words : amateur fishing, fishing administration, shad, Rhône, sociology. 


\section{INTRODUCTION}

L'alose, poisson migrateur amphihalin qui colonise le Rhône chaque printemps, fait l'objet depuis la fin des années 1980 d'une politique de réhabilitation'. Les barrages de la Compagnie Nationale du Rhône établis sur le fleuve ont eu pour effet de restreindre les zones de migration de cette espèce qui frayait le long du cours d'eau au niveau de ses divers affluents. En 1993, une association Migrateurs Rhône-Méditerranée a été créée pour remédier à ce dysfonctionnement regroupant en son sein des scientifiques experts de la question des poissons migrateurs et les principaux gestionnaires à la fois du Rhône, de la pêche amateur ti professionnelle et des milieux piscicoles dans leur ensemble. L'alose s'est donc vu dotée de qualités nouvelles, à savoir d'être l'espèce piscicole garante de la restauration du Rhône; le symbole d'un fleuve nouveau où la migration naturelle aurait repris ses droits sur la mainmise du milieu par l'homme (BARTHÉLÉMY, 1999).

L'association est ainsi devenue un nouvel acteur de la gestion piscicole du fleuve. Elle met en place différents types de suivis scientifiques visant à rendre compte de l'état et de l'évolution de la migration. Ainsi, les captures réalisées par les pêcheurs amateurs peuvent servir d'indicateurs. Dans la région du Bas-Rhône, une quinzaine d'individus, concentrés en aval de l'usine-écluse de Vallabrègues sur le canal de fuite, pratiquent chaque année la pêche au filet fixe. Leur pratique de pêche régulière dans la saison et leurs prises pouvant être facilement comptabilisées, les responsables de l'association ont pensé que le suivi de cette pêcherie pourrait apporter des données relativement fiables sur la migration. C'est à ce titte que l'association Migrateurs Rhône-Méditerranée m'a demandée de réaliser une enquểle sociologique auprès de ces pêcheurs afin de décrire leurs pratjques de pêche et de cernerles atouts et les contraintes pour mettre en place ce suivi (BARTHÉLÉMY, 1995). Depuis 1996, celui-ci est effectivement réalisé, nécessitant la présence d'une personne de l'association auprès des pêcheurs durant la saison de pêche.

Notre enquête a certainement jeté les bases de cette collaboration mais elle a aussi révélé des antagonismes sociaux entre les pêcheurs amateurs et les gestionnaires. Le propos de cet article n'est pas de relater les préjugés véhiculés des uns envers les autres (en accentuant un peu, le pêcheur n'est qu'un braconnier irresponsable pour le gestionnaire etce dernier, un technocrate incompétent pour ce premier!) mais essayer d'analyser ce malentendu fréquemment observable autour de la gestion des milieux piscicoles. En effet, la sauvegarde des poissons migrateurs portée par des politiques de gestion dite écologique pose all sociologue la question suivante : quelle est la légitimité de ce type d'actions par rapportaux usagers quotidiens des espaces concernés?

\section{CADRE THÉORIQUE ET EMPIRIQUE DE LA RECHERCHE}

Les relations entretenues autour de l'alose par les pêcheurs aux engins et les gestionnaires peuvent être analysées comme l'appropriation du Rhône par deux groupes sociaux distincts. En effet, la sociologie de l'environnement, dont le principal objet d'analyse concerne les relations entre la société et la nature, étudie les pratiques de groupes sociaux différents par rapport aux ressources naturelles. Ainsi, le pêcheur amateur s'inscrit-il dans une relation de prédation par rapport au fleuve; le gestionnaire, quant à lui, étudie, gère of

\footnotetext{
1. Entre 1989 et 1991, le Ministère de l'Environnement a instauré les Schémas Départementaux de Vocation Piscicle (SDVP) qui devaient définir les orientations pour la restauration et la mise en ceuvre du patrimoine piscicole la gestion des poissons migrateurs devient alors une priorité nationale. Deux plans nationaux se succéderont : leplan Migrateurs de 1981 à 1986 et le plan Retour aux Sources de 1991 à 1995.
} 
applique des mesures de protection par rapport à l'alose. Ces relations pratiques à l'espace s'accompagnent de la part des individus d'un ensemble d'argumentaires, de connaissances et de points de vue qui viennent légitimer leur appropriation de la nature (CADORET, 1985 ; MATHIEU et JOLLIVET, 1989). Ceux-ci sont souvent mis en exergue lors de conflits d'usage. Le travail du sociologue consiste alors à étudier ces pratiques conflictuelles à travers plusieurs méthodes.

Tout d'abord, les arguments de chacun des groupes sociaux peuvent être analysés. Cette démarche consiste à prendre en compte le sens donné par les individus à leurs actes. Celle-ci s'inscrit dans une sociologie dite compréhensive qui tente de comprendre l'action des hommes du point de vue de leur subjectivité et de leurs valeurs (WEBER, 1922, 1971). Laction sociale résulte des décisions prises par des individus qui donnent eux-mêmes un sens à leur action. La méthode de travail a été l'enquête de terrain auprès des pêcheurs amateurs. Celle-ci consiste à l'observation des pêcheurs sur le lieu de pêche et au recueil de leurs discours par la technique de l'entretien semi-directif. Celui-ci repose sur un choix de questions dites ouvertes qui permettent aux individus de s'entretenir librement d'un sujet. Les diverses personnes qui composent l'association Migrateurs Rhône-Méditerranée ont été interrogées d'après la même technique. Les discours ainsi recueillis sont ensuite intégralement retranscrits et étudiés.

Un groupe social peut aussi se lire à travers les caractéristiques socio-économiques des individus qui le composent : le sexe, l'âge, la profession exercée, le niveau de diplômes ou de qualification professionnelle... La démarche sociologique dite déterministe repose sur la mise en relation causale entre ces diverses caractéristiques et les attitudes ou les comportements observés des individus. Ainsi, les travaux de Plerre Bourdieu ont montré comment, par exemple, les pratiques de consommation sont gouvernées par des goûts socialement définis par un habitus de classe. Celui-ci est un principe qui structure la perception et laction des individus selon leur appartenance sociale. Ainsi, plus les individus appartiennent à des groupes sociaux semblables, plus leurs habitus sont communs : « La connaissance pratique du monde social que suppose la conduite "raisonnable" dans ce monde met en ceuvre des schemes classificatoires (...), schèmes historiques de perception et d'appréciation qui sont le produit de la division objective en classes (classes d'âge, classes sexuelles, classes sociales) et qui fonctionnent en deçà de la conscience et du discours. Étant le produit de lincorporation des structures fondamentales d'une société, ces principes de division sont communs à l'ensemble des agents de cette société et rendent possibles la production d'un monde commun et sensé, d'un monde de sens commun "(BOURDIEU, 1979).

Ainsi, les pêcheurs amateurs et les gestionnaires de l'association se distinguent non seulement par rapport à leurs pratiques et leurs représentations du milieu fluvial mais aussi par rapport au milieu social dans lequel ils évoluent.

\section{LES PÊCHEURS D'ALOSES AU CARRELET : POUR UNE NATURE À CONSOMMER SANS MODÉRATION}

La pêche de l'alose consiste à manceuvrer un filet de $25 \mathrm{~m}^{2}$ de superficie suspendu à un mât. $\dot{A}$ Vallabrègues, le carrelet est fixé sur une barque elle-même reliée à la berge par une simple passerelle de bois ou par une autre barque selon le niveau de l'eau. Cette technique de pêche est simple et rudimentaire. Le geste du pêcheur consiste à baisser et relever le filet et lors de prises, il s'arme d'une épuisette pour récupérer les poissons et les déposer dans une
caisse. 
Le choix du site de pêche est lié à la présence du premier grand obstacle que rencontrent les aloses dans leur remontée. Quatorze pêcheurs pratiquaient ce type de pêche, près de leur lieu d'habitation, avant les travaux de la Compagnie Nationale du Rhône. En eftet, ce sont essentiellement des retraités. La moyenne d'âge pour les 22 pêcheurs rencontrés durant la saison de pêche 1995 est de 62 ans. Ils font donc partis d'une génération qui pratiquait le fleuve avant sa canalisation. Aujourd'hui, ce sont eux qui migrent à la place des aloses! La pêche de l'alose (13 pêcheurs sur 22) mais aussi la pêche aux engins de manière plus générale est essentiellement exercée dans le département du Gard, sur la rive droitedu fleuve. Cette préférence peut s'expliquer par la présence des affluents Cévenols et Gardois (Ardèche, Cèze, Gard) plus chauds et donc plus concernés par la migration des aloses que les affluents préalpins (Durance, Ouvèze...) (QUIGNARD, 1978).

Une autre variable explicative à cette provenance géographique des pêcheurs aux engins réside dans l'appartenance au monde rural. Cela s'illustre dans les professions exercées par les pêcheurs. Ainsi, sur 22, 6 sont ou étaient exploitants agricoles, 10 ouvriers $t$ 6 employés. La part des agriculteurs est supérieure à la moyenne nationale. Ainsi, sur 100 pêcheurs amateurs en France, 3 sont exploitants agricoles, 11 employés et 32 sont ouvriers (PICON, 1991). Ainsi, la part des ouvriers est importante aussi bien pour la pêche de l'alose que pour tous les types de pêche confondus. La pêche de l'alose est populaire (parlak, nous entendons des niveaux de diplômes équivalents au certificat d'études et des emplois d'ouvriers ou d'employés) et rurale (par la présence d'agriculteurs et de pêcheurs habitant dans des communes de moins de 5000 habitants). Cette prégnance du monde rural explique en partie la relation entretenue par les pêcheurs avec l'alose.

En effet, celle-ci est uniquement recherchée pour sa chair. Les pêcheurs sont des amateurs de l'alose préparée à l'oseille ou au vin blanc. La pêche au carrelet permet de se constituer une réserve pour l'année à venir. Lorsque le poisson est abondant, le pêcheurle donne, le vend ou le troque. Un marché illicite prend place chaque année au bord du Rhône. Amis de pêcheurs ou clients anonymes traînent près des bateaux pour acheter quelques poissons. L'argent n'est pas toujours la monnaie d'échanges; il est parfois remplacé par des œufs, du vin, des légumes... L'alose est donc, pour ces pêcheurs, un aliment consommable que le fleuve propose. Celui-ci est perçu comme une nature - ressource, c'est-à-dire comme un milieu naturel riche et abondant. Le rapport à la nature se construit dans une relation classique de prédation. Les pêcheurs d'aloses prônent une alimentation presque vẹcue en autarcie. Ils critiquent la consommation de masse qui éloigne le consommateur du producteur: Eux jardinent, chassent, pêchent et cultivent. La vente ou l'échange des aloses est dane compatible avec ce système de pensée qui fait de la nature, un milieu généreux offrant des produits de qualité dont il faut user.

Cette volonté de ne consommer que ce que l'on cueille, chasse ou pêche est un that constitutif de la relation entretenue entre les pêcheurs et le milieu piscicole. II se retrowe sous une autre forme, à travers le matériel de pêche. Seuls le bateau, les filets et les câbles sont achetés par les pêcheurs. Le reste de l'équipement qui comprend entre autre le mât etlla système de poulie est fabriqué avec des matériaux de récupération. La manivelle qui permet de lever et de baisser le filet est composé d'un pédalier de vélo et d'un volant de 2 CV. La paith du bricolage est importante et elle traduit le volonté de ne pas dépenser beaucoup diargent. dans le cadre d'un loisir. Bien au contraire, le temps passé à ne pas travailler doit rappontar quelque chose pour manger, échanger ou posséder. Le loisir est lié à la notion de nécessitê pas obligatoirement matérielle mais surtout sociale : "La "nécessité " est aussi une nécessité sociale liée à l'image dans le milieu social... Il s'agit de respecter la norme discours de la polyvalence tient à une définition de l'excellence virile et de la responsabithte assumée dans la vie familiale " (BONETTE-LUCAT, 1991). Pour les pêcheurs d'aloses lt loisir est lié à une notion de travall et de production. 
Pourtant, cet aspect laborieux de la pêche amateur ne doit pas occulter son aspect ludique. Lorsque l'on demande aux pêcheurs ce qui les motivent le plus dans la pratique de cette pêche, ils répondent le contact avec la nature et la convivialité. La nature représente un espace socialement différent de l'espace domestique ou de l'espace du travail. Au bord du Rhône, les pêcheurs se retrouvent entre hommes loin de leurs responsabilités quotidiennes. certains construisent des abris de fortune réalisés avec des matériaux de récupération (planches, tôles, fauteuils, bancs, tables...) où se tiennent les repas. En effet, la journée de pêche est rythmée par le déjeuner copieux et bien arrosé. Les pêcheurs peuvent faire griller des aloses mais aussi de la viande ou réchauffer des plats préparés à la maison. La pêche de l'alose est bien loin de l'image du pêcheur solitaire et silencieux. Cette convivialité repose sur une pratique collective de la pêche. Sur les 14 bateaux observés, 5 seulement sont exploités par un seul pêcheur. La majorité des bateaux sont l'affaire de deux ou trois amis qui partagent les frais de licence et d'entretien. Certains forment des équipes (de 5 à 6 pêcheurs) qui pêchent l'alose ensemble puis qui se retrouvent pour la chasse et parfois même entretiennent des relations professionnelles (pour les exploitants agricoles, par exemple). Le temps passé sur le fleuve pour pêcher l'alose correspond pour les pêcheurs à un temps de rupture par rapport au quotidien. C'est un lieu essentiellement masculin où l'on se retrouve entre amis, où la parole et les gestes sont libérés de certaines contraintes sociales.

Ce sentiment d'être libre et maître de soi implique une forte appropriation de l'espace fluvial. Chaque pêcheur délimite son territoire de pêche et il lui est difficile d'accepter les interdictions ou des réglementations provenant du propriétaire des lieux très facilement oublié.

Les pêcheurs d'aloses au carrelet forment donc un groupe social issu du monde rural, agricole et populaire. Leurs relations au milieu piscicole reposent sur trois principes essentiels : l'alose est un poisson aliment qui doit être consommé ; le loisir est associé à des valeurs de travail, donc, la pêche se doit de rapporter un bien (ce qui justifie la vente et donc le braconnage du poisson) ; enfin, la vie sociale autour du cabanon au bord du Rhône marque un temps libérateur. Nous pouvons donc nous demander comment ces pêcheurs ont accueilli la présence, les actions et les principes portés par l'association Migrateurs RhôneMéditerranée.

\section{LA QUESTION DES SUIVIS SCIENTIFIQUES : DES DÉSACCORDS ISSUS DE FORMES DE CONNAISSANCES DIFFÉRENTES}

Cette association et les membres, qui la composent, représentent les gestionnairesprotecteurs des poissons migrateurs du Rhône ${ }^{z}$. Le fleuve est perçu comme un espace meurtri et appauvri par la présence des aménagements de la Compagnie Nationale du Rhône : Autrefois, tous ces poissons (migrateurs) étaient abondants dans les eaux du bassin du Rhône. Mais, le fleuve et ses affluents ont été domestiqués, des barrages ont été construits. Les poissons se sont vus alors interdire l'accès à leurs zones de reproduction. Leur survie était menacée. Heureusement, conscients de ce patrimoine écologique qui disparaissait, les hommes ont décidé de venir à leur secours " (texte issue d'une plaquette de présentation de l'association). Pour remédier à cette situation jugée catastrophique, lassociation a recours à plusieurs types d'actions dont la recherche scientifique et le réaménagement du cours d'eau et des équipements existants pour permettre la migration des

\footnotetext{
- En 1998, 26 organismes étalent impliqués de manière différente dans l'association, lls peuvent se répartir en quatre ¿î́gories: le milieu associatif dominé par les associations de pêcheurs amateurs, les collectivités locales, le monde s.ientifique (INRA, CNRS...) et les institutions administratives (CNR, Agence de l'Eau, Ministère de l'Environnement, onseil Supérieur de la Pêche, Electricité de France...).
} 
aloses. Toute une littérature technique et scientifique est donc produite sur l'alose du Rhône (biologie, génétique, connaissances de la migration), sur les sites de frayères (qualité de l'eau, demande d'arrêtés de biotope) et sur les aménagements (construction de passes à poisson...). Pratiques et savoirs techniques ou scientifiques sont indéniablement liés. Pour les gestionnaires, les données technico-scientifiques basées sur un savoir écologique garantissent la meilleure gestion à appliquer (FABIANI, 1995 ; MICOUD, 1993).

De plus, leur représentation de la nature est liée à un mode de pensée écosystémique. Celui-ci se traduit par l'idée que tous les éléments d'un milieu sont en interdépendance. Ainsi, les projets de l'association reposent sur le fait que si l'alose arrive à franchir les barrages, les autres poissons migrateurs pourront le faire mais cela pourra aussi bénéficier aux autres espèces piscicoles. Si l'alose arrive à frayer sur certains sites, cela prouve que l'eau est d'une qualité honorable. En fin de compte, par l'alose, c'est une nature sauvée et restaurée qui s'annonce. De plus, les acteurs prévoient un développement économique et social induit parle retour des poissons migrateurs. L'homme est alors réintroduit dans l'écosystème par les pratiques même de la gestion écologique ou par des pratiques " acceptées ", commela pêche professionnelle et la pêche amateur (associée à des projets de développement touristique). Chantal Aspe a étudié ce mode de pensée par rapport à certaines pratiques innovantes concernant la gestion des eaux usées : « La construction de cette représentation s'organise dans le passage de la nature désignée comme ressource, à la nature désignée comme patrimoine. Cette notion de patrimoine prend sens dans l'idée de transmission, d'interdépendance entre les actions présentes et futures, de relation systémique dans le rapport nature/société. C'est dans ce système de pensée que se réalisent les pratiques innovantes des élus locaux en matière de traitement des eaux usées. Ces élus ne considerent pas l'épuration comme une fin en soi mais comme le maillon d'une chaîne et pensent en termes d'interactions entre économie locale, protection de l'environnement, développement local et production nouvelle des ressources naturelles "(ASPE, 1991). Ainsi, l'association gestionnaire se caractérise par un savoir technico-scientifique lié à une représentation systémique du milieu piscicole rhodanien.

Nous pouvons donc mieux comprendre la différence de perceptions des populations d'aloses entre gestionnaires et pêcheurs au carrelet. Ces derniers ne sont cependant pas totalement détachés d'une vision écologiste de la migration. Ils ont effectivement observé sa régression mais ils n'associent absolument pas celle-ci à l'image d'un fleuve si appauvri qu'lls devraient, pour cela, remettre en cause leurs pratiques de pêche.

Le suivi scientifique instauré depuis quelques années fonctionne car une personne de l'association est chargée de collecter les données, auprès des pêcheurs, durant toute la saison de pêche. Cependant, l'idéal pour les scientifiques serait que ces derniers remplissent eux-mêmes un carnet. Or, un carnet de pêche existe déjà et il est délivré à chaque pêcheur, chaque année, par le Service de la Navigation pour l'ensemble du Rhône. Les données sont ensuite étudiées par le Conseil Supérieur de la Pêche. La plupart des pêcheurs d'aloses disent ne pas le remplir. Ce blocage dans la restitution des prises trouve des explications dans la description faite auparavant de la pêcherie. Le fait de devoir déclarer ses prises est perçu comme une atteinte à la liberté qui est le sentiment exprimé par les pêcheurs au bord du Rhône. C'est imposé une contrainte là où justement, on les contourne.

Un problème réside aussi dans le devenir des données. En effet, les pêcheurs sont rarement tenus au courant de ce qui a été produit ou réalisé grâce à leurs déclarations. L $\ell$ principe du carnet de pêche reste un outil administratif qui n'établit aucun contact entre les pêcheurs et les gestionnaires. De plus, il est nécessaire d'écrire pour tenir ce carnet alors que 
les pêcheurs ne veulent surtout pas de contrainte d'ordre administratif dans l'exercice de leur loisir. Le comptage, les statistiques font partie de ce savoir technico-scientifique décrit précédemment et qui n'est pas celui détenu par les pêcheurs. Ceux-ci détiennent un ensemble de connaissances qui reposent essentiellement sur l'observation de l'état du fleuve, répétée depuis de nombreuses années. Ainsi, le pêcheur observe la turbidité de l'eau (l'eau idéale est trouble ou brouillée qui permet le déplacement des aloses et cache le filet) et il peut reconnaître la présence des affluents qui se déversent à un instant donné en abondance dans le Rhône. Ces derniers ont une influence majeure sur la présence des aloses (l'lsère est noire, sale et froide et n'est pas favorable alors que la Saône est jugée excellente). Ces connaissances constituent un atout pour les pêcheurs qui justifient ainsi leur appropriation du fleuve et les fait rester parfois dubitatifs quant aux expériences scientifiques menées sous leurs yeux durant la saison de pêche.

\section{CONCLUSION : DE LA LÉGITIMITÉ RECHERCHÉE PAR LES UNS ET PAR LES AUTRES...}

Sans conflits réels, les pêcheurs et les gestionnaires de l'alose du Rhône ont trouvé un compromis à travers la mise en place d'un suivi scientifique. Alors que les membres de l'association espèrent que les pêcheurs remplissent de manière autonome et fiable un carnet ; les pêcheurs voudraient que leurs désirs de liberté au bord du Rhône soient respectés et non entravés par de nouvelles réglementations.

Cet exemple auprès des pêcheurs d'aloses rhodaniens nous éclaire sur la difficile légitimation des politiques de gestion piscicole des poissons migrateurs auprès des pêcheurs amateurs. En sociologie, il est classique de se référer à Max Weber quant à cette notion de légitimité. Celle-ci renvoie à la question du pouvoir car poser le problème de la légitimation, ciest savoir comment un pouvoir se rend acceptable. Max Weber distingue trois formes de domination et de légitimité (WEBER, (1922), 1971) : la domination traditionnelle qui fonde sa légitimité sur le caractère sacré de la tradition (le pouvoir patriarcal au sein de la famille) ; la domination charismatique, issue d'une personnalité dotée d'une aura exceptionnelle (prophètes, dictateurs) ; la domination rationnelle ef légale qui s'appuie sur la légitimation d'un droit abstrait et impersonnel. Le pouvoir se justifie ainsi par la compétence et la rationalité des choix.

Ainsi, les pêcheurs d'aloses légitiment leur présence par rapport à leurs années dexpérience, à leurs savoirs naturalistes et à l'aspect résiduel de ce type de pêche. Leur lègitimité serait donc plutôt du registre du «traditionnel ». Pour les gestionnaires, ils fondent leur pouvoir sur leur compétence scientifique, la rationalité de leurs pratiques de gestion et de protection et la mise en place de nouvelles législations. Cette légitimité du type rationnelle et légale ne s'impose pas auprès des pêcheurs aux engins. L'association va donc puiser dans une autre forme de légitimité, la légitimité charismatique à travers les espoirs fondés en lécologie, comme une nouvelle voie économiquement et socialement prometteuse.

Mais, celle-ci ne trouve que très peu d'échos auprès des pêcheurs d'aloses pour qui lécologie n'est pas sacrée et pour qui le discours technico-scientifique reste trop administratif. De l'autre, les scientifiques restent sceptiques quant à la pertinence des connaissances acquises par les pêcheurs et modérés quant à accepter leurs pratiques de braconnage. En fin de compte, aucun des deux groupes sociaux n'arrive parfaitement à imposer son point de vue dans cette gestion piscicole car aucun des deux ne peut encore asseoir son pouvoir. Si, autour du Rhône, se diffuse un discours faisant du retour de l'alose, l'emblème d'un gestion écologique réussie ; l'observation de la mise en pratique de cette gestion laisse entrevoir diverses formes d'appropriation du fleuve, entre Nature populaire et Nature savante. 


\section{REMERCIEMENTS}

L'auteur tient à remercier l'association Migrateurs Rhône-Méditerranée et particulièrement Jean-Yves Menella pour lui avoir confié cette étude sociologique et lavoir ainsi soutenu dans le cadre d'un doctorat de Sociologie sur les pratiques de pêche amateur dans la région du Bas-Rhône.

\section{BIBLIOGRAPHIE}

Actes du séminaire de Sommières, 1983. Les savoirs naturalistes populaires, Édition dela Maison des Sciences de l'Homme, Paris, 94 p.

ASPE C., 1991. L'environnement: une histoire entre les couches moyennes et la localité. In: Chercheurs d'eau en Méditerranée, Éditions du Félin, Paris, 191-213.

BARTHÉLÉMY C., 1995. À l'abri des acacias en fleur... les pêcheurs d'aloses du Rhône. Étude interdisciplinaire des pratiques de pëche amateur aux engins dans la région du Bas-Rhône, Rapport Association Migrateurs Rhône-Méditerranée, 101 p.

BARTHÉLÉMY C., 1999. L'alose, quel symbole pour quel fleuve restauré? Le poisson migrateur, porte-parole de la patrimonialisation du Rhône. Rapport Patrimonialisation du vivant et structuration de l'espace, Région Rhône-Alpes, Agence Rhône-Alpes pour les sciences sociales et humaines, 32-50.

BONETTE-LUCAT C., 1991. Les bricoleurs : entre polyvalence et spécialisation. Sociétés contemporaines, 8, 61-85.

BOURDIEU P., 1979. La distinction, critique sociale du jugement, Les Éditions de Minuit, Paris, $670 \mathrm{p}$.

CADORET A. (ss la direction de), 1985. Protection de la nature. Histoire et idéologie. Dela nature à l'environnement, L'Harmattan, Paris, $245 \mathrm{p}$.

FABIANI J., 1995. Les recréateurs de nature. Enjeu et justification d'une pratique paradoxale. Natures - Sciences - Sociétés Volume 3, Hors Série, 85-97.

MATHIEU N., JOLLIVET M. (ss la direction de), 1989. Du rural à l'environnement. La question de la nature aujourd'hui, Association des Ruralistes Français, L'Harmattan, Paris, $352 \mathrm{p}$.

MICOUD A., 1993. Vers un nouvel animal sauvage : le sauvage « naturalisé vivant "? Natures, Sciences, Sociétés Vol. 1, 3, 203-210.

PICON B., 1991. Chasse, pêche, cueillette : un même objet support d'attitudes et de pratiques sociales différenciées. Sociétés contemporaines, 8, 87-100.

QUIGNARD J., 1978. Le Rhône et quelques problèmes concernant ses poissons ou histoire non naturelle de ce fleuve, Éditions de la Sabranenque, Nîmes, 125 p.

WEBER M., (1922), 1971. Économie et société, Plon, Paris. 